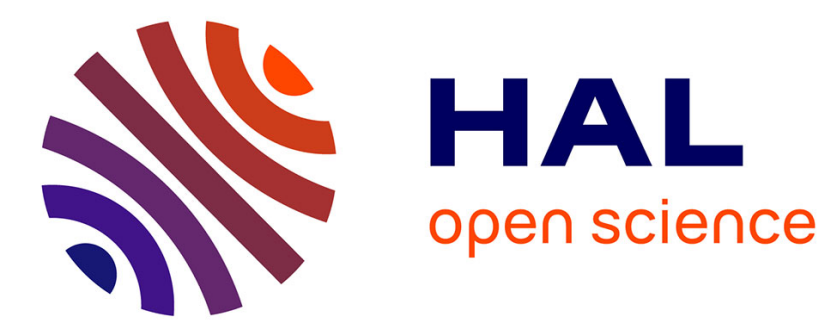

\title{
Dynamic spin-correlation function near the antiferromagnetic quantum phase transition of heavy fermions \\ C. Pépin, M. Lavagna
}

\section{- To cite this version:}

C. Pépin, M. Lavagna. Dynamic spin-correlation function near the antiferromagnetic quantum phase transition of heavy fermions. Physical Review B: Condensed Matter and Materials Physics (19982015), 1999, 59 (4), pp.2591 - 2598. 10.1103/PhysRevB.59.2591 . hal-01896148

\author{
HAL Id: hal-01896148 \\ https://hal.science/hal-01896148
}

Submitted on 16 Oct 2018

HAL is a multi-disciplinary open access archive for the deposit and dissemination of scientific research documents, whether they are published or not. The documents may come from teaching and research institutions in France or abroad, or from public or private research centers.
L'archive ouverte pluridisciplinaire HAL, est destinée au dépôt et à la diffusion de documents scientifiques de niveau recherche, publiés ou non, émanant des établissements d'enseignement et de recherche français ou étrangers, des laboratoires publics ou privés. 


\title{
Dynamic spin-correlation function near the antiferromagnetic quantum phase transition of heavy fermions
}

\author{
C. Pépin* and M. Lavagna ${ }^{\dagger}$ \\ Commissariat à l'Energie Atomique, Département de Recherche Fondamentale sur la Matière Condensée/SPSMS, \\ 17, rue des Martyrs, 38054 Grenoble Cedex 9, France
}

(Received 31 March 1998)

\begin{abstract}
The dynamical spin susceptibility is studied in the magnetically disordered phase of heavy-Fermion systems near the antiferromagnetic quantum phase transition. In the framework of the $S=1 / 2 \mathrm{Kondo}$ lattice model, we introduce a perturbative expansion treating the spin and Kondo-like degrees of freedom on an equal footing. The dynamical spin susceptibility displays a two-component behavior in agreement with the inelastic neutron scattering (INS) experiments performed in $\mathrm{CeCu}_{6}, \mathrm{Ce}_{1-x} \mathrm{La}_{x} \mathrm{Ru}_{2} \mathrm{Si}_{2}$, and $\mathrm{UPt}_{3}$ : a quasielastic $q$-independent peak as in a Fermi-liquid theory, and a strongly $q$-dependent inelastic peak typical of a non-Fermi-liquid behavior. Very strikingly, the position of the inelastic peak is found to be pushed to zero at the antiferromagnetic transition. The picture is consistent with the neutron cross sections observed in INS experiments. [S0163-1829(99)13603-8]
\end{abstract}

\section{INTRODUCTION}

One of the most striking properties of heavy fermion compounds discovered these last years is the existence of a quantum phase transition ${ }^{1,2}$ driven by composition change (at $x_{C}=0.1$ in $\mathrm{CeCu}_{6-x} \mathrm{Au}_{x}$ and $x_{C}=0.08$ in $\left.\mathrm{Ce}_{1-x} \mathrm{La}_{x} \mathrm{Ru}_{2} \mathrm{Si}_{2}\right)$, pressure or magnetic field. It has been largely discussed in various theoretical approaches. ${ }^{5,6}$ Important insight is provided by the evolution of the low-temperature neutron cross section measured by inelastic neutron scattering (INS) experiments when getting closer to the magnetic instability. The experiments performed in pure compounds $\mathrm{CeCu}_{6}$ and $\mathrm{CeRu}_{2} \mathrm{Si}_{2}$ by Regnault et al. ${ }^{3}$ and Aeppli et al. ${ }^{4}$ have shown the presence of two distinct contributions to the dynamic magnetic structure factor: a q-independent quasielastic component, and a strongly q-dependent inelastic peak with a maximum at the value $\omega_{\max }$ of the frequency. The former corresponds to localized excitations of Kondo-type while the latter peaked at some wave vector $\mathbf{Q}$ is believed to be associated with intersite magnetic correlations due to RudermanKittel-Kasuya-Yosida (RKKY) interactions. The frequency width of the quasielastic and inelastic peaks respectively define the single-site and intersite relaxation rate $\Gamma_{S S}$ and $\Gamma_{I S}$. Such features have also been observed in $\mathrm{UPt}_{3}$ and called as "slow" and "fast" components by Bernhoeft and Lonzarich. ${ }^{7}$ Later on, INS experiments have been performed with varying compositions as in $\mathrm{Ce}_{1-x} \mathrm{La}_{x} \mathrm{Ru}_{2} \mathrm{Si}_{2} .{ }^{8}$ It has been observed a narrowing of the single-site relaxation rate $\Gamma_{S S}$ when getting closer to the magnetic transition. At the same time, both the position of the inelastic peak $\omega_{\max }$ and the intersite relaxation rate $\Gamma_{I S}$ drastically decrease when getting near the magnetic instability. Table I reports the values of $\Gamma_{S S}, \Gamma_{I S}$, and $\omega_{\max }$ for the different compounds.

Any theory aimed to describe the quantum critical phenomena in heavy-fermion compounds should account for the so-quoted behavior of the dynamical spin susceptibility. We start from the Kondo-lattice model, which is believed to describe the physics of these systems. We refer to the recent paper of Tsunetsugu et al. ${ }^{9}$ for a review of the model. As already pointed out by Doniach in his initial paper, ${ }^{10}$ the main features result of the competition between the Kondo effect and the RKKY interactions among spins mediated by the conduction electrons. Most of the theories developed so far $^{11-15}$ agree with the existence of a hybridization gap, which splits the Abrikosov-Suhl or Kondo resonance formed at the Fermi level. The role of the interband transitions has been outlined for long in order to explain the inelastic component of the dynamical spin susceptibility. For instance, the theories based on a $1 / N$ expansion ${ }^{16-20}$ (where $N$ is simultaneously the degeneracy of the conduction electrons and of the spin channels) predict a maximum of $\chi^{\prime \prime}\left(k_{F}, \omega\right) / \omega$ at $\omega_{\max }$ of the order of the indirect hybridization gap. ${ }^{21}$ However, the $1 / N$-expansion theories present serious drawbacks: (i) the spin-fluctuation effects are automatically ruled out since the RKKY interactions only occur at the following order in $1 / N^{2},{ }^{22}$ (ii) they then fail to describe any magnetic instability and hence the quantum critical phenomena mentioned above, and (iii) the predictions for $\omega_{\max }$ and the associated relaxation rate cannot account for the experimental observations near the magnetic instability. An improvement brought by Doniach ${ }^{23}$ consists to consider the $1 / N^{2}$ corrections in an instantaneous approximation: it gives back the ladder diagram contribution to the dynamical spin susceptibility and then accounts for the spin-fluctuation effects. Other approaches were proposed in Refs. 24-26. But still the predictions for the frequency dependence of the dynamic

TABLE I. Values of the single-site and intersite relaxation rates $\Gamma_{S S}$ and $\Gamma_{I S}$ and position of the inelastic peak $\omega_{\max }$ extracted from the inelastic neutron scattering (INS) measurements performed in $\mathrm{CeCu}_{6}$ (Ref. 3) and $\mathrm{Ce}_{1-x} \mathrm{La}_{x} \mathrm{Ru}_{2} \mathrm{Si}_{2}$ at $x=0$ and $x=0.075$ (Ref. 8).

\begin{tabular}{lccc}
\hline \hline & $\Gamma_{S S}(\mathrm{meV})$ & $\Gamma_{I S}(\mathrm{meV})$ & $\omega_{\max }(\mathrm{meV})$ \\
\hline $\mathrm{CeCu}_{6}$ & 0.42 & 0.2 & 0.25 \\
$\mathrm{CeRu}_{2} \mathrm{Si}_{2}$ & 2.0 & 0.75 & 1.2 \\
$\mathrm{Ce}_{1-x} \mathrm{La}_{x} \mathrm{Ru}_{2} \mathrm{Si}_{2}$ & 1.4 & 0.2 & 0.2 \\
\hline \hline
\end{tabular}


magnetic structure factor presents a gap of the order of the hybridization gap whatever the value of the interaction is. On the other hand, in front of the difficulties encountered when starting from microscopic descriptions, various phenomenological models (as the duality model of Kuramoto and Miyake $^{27,28}$ and Ref. 7) have been introduced to describe both the spin fluctuation and the itinerant electron aspects with some successful predictions as the weak antiferromagnetism of these systems.

In this paper, we develop a systematic approach to the Kondo-lattice model for $S=1 / 2(N=2)$ in which the Kondolike and the spin degrees of freedom are treated on an equal footing. The presented approach shows some similarities with earlier works. ${ }^{11,15}$ But while Refs. 11 and 15 essentially describe the phase diagram of the Kondo lattice at a meanfield level, we focus on the effects of spin fluctuations in the magnetically disordered phase hence bringing the spinfluctuation and the Kondo-effect theories together. The saddle-point results and the Gaussian fluctuations in the charge channel are consistent with the standard $1 / N$ theories. In addition, the Gaussian fluctuations in the spin channel restore the spin-fluctuation effects that were missing in the $1 / N$ expansion. The general expression of the dynamical spin susceptibility that we derive reproduces some of the features postulated in the phenomenological models. It presents a two-component behavior: a quasielastic component superimposed on an inelastic peak with renormalized values of the relaxation rates, susceptibilities and $\omega_{\max }$. In a very striking way, $\omega_{\max }$ is pushed to zero and the inelastic mode becomes soft at the antiferromagnetic phase transition with vanishing relaxation rate. Predictions are quantitatively compared with experimental results. The quasielastic peak is typical of a Fermi liquid while the other mode breaks the Fermi-liquid description. Our approach might offer new prospects for the study of the quantum critical phenomena in the vicinity of the antiferromagnetic phase transition.

\section{PRESENTATION OF THE APPROACH}

We consider the Kondo-lattice model (KLM) constituted by a periodic array of Kondo impurities with an average number of conduction electrons per site $n_{c}<1$. In the grand canonical ensemble, the Hamiltonian is written as

$$
\begin{aligned}
H= & \sum_{k \sigma} \varepsilon_{k} c_{k \sigma}^{\dagger} c_{k \sigma}+J \sum_{i} \mathbf{S}_{i} \cdot \sum_{\sigma \sigma^{\prime}} c_{i \sigma}^{\dagger} \boldsymbol{\sigma}_{\sigma \sigma^{\prime}} c_{i \sigma^{\prime}} \\
& -\mu N_{S}\left(\frac{1}{N_{S}} \sum_{k \sigma} c_{k \sigma}^{\dagger} c_{k \sigma}-n_{c}\right)
\end{aligned}
$$

in which $\mathbf{S}_{i}$ represents the spin $(S=1 / 2)$ of the impurities distributed on the sites (in number $N_{S}$ ) of a periodic lattice; $c_{k \sigma}^{\dagger}$ is the creation operator of the conduction electron of momentum $\mathbf{k}$, spin quantum number $\sigma$ characterized by the energy $\boldsymbol{\epsilon}_{k}=-\Sigma_{\langle i, j\rangle} t_{i j} \exp \left(i \mathbf{k} \cdot \mathbf{R}_{i j}\right)$ and the chemical potential $\mu ; \boldsymbol{\tau}$ are the Pauli matrices $\left(\boldsymbol{\tau}^{x}, \boldsymbol{\tau}^{y}, \boldsymbol{\tau}^{z}\right)$ and $\boldsymbol{\tau}^{0}$ the unit matrix; $J$ is the antiferromagnetic Kondo interaction $(J>0)$.

We use the Abrikosov pseudofermion representation of the spin $\mathbf{S}_{i}: \mathbf{S}_{i}=\Sigma_{\sigma \sigma^{\prime}} f_{i \sigma}^{\dagger} \boldsymbol{\tau}_{\sigma \sigma^{\prime}} f_{i \sigma^{\prime}}$. The projection into the physical subspace is implemented by a local constraint

$$
Q_{i}=\sum_{\sigma} f_{i \sigma}^{+} f_{i \sigma}-1=0
$$

A Lagrange multiplier $\lambda_{i}$ is introduced to enforce the local constraint $Q_{i}$. Since $\left[Q_{i}, H\right]=0, \lambda_{i}$ is time independent.

In this representation, the partition function of the KLM can be expressed as a functional integral over the coherent states of the fermion fields

$$
Z=\int \mathcal{D} c_{i \sigma} \mathcal{D} f_{i \sigma} d \lambda_{i} \exp \left[-\int_{0}^{\beta} \mathcal{L}(\tau) d \tau\right],
$$

where the Lagrangian $\mathcal{L}(\tau)$ is given by

$$
\begin{gathered}
\mathcal{L}(\tau)=\mathcal{L}_{0}(\tau)+H_{0}(\tau)+H_{J}(\tau), \\
\mathcal{L}_{0}(\tau)=\sum_{i \sigma} c_{i \sigma}^{\dagger} \partial_{\tau} c_{i \sigma}+f_{i \sigma}^{\dagger} \partial_{\tau} f_{i \sigma}, \\
H_{0}(\tau)=\sum_{k \sigma} \epsilon_{k} c_{k \sigma}^{\dagger} c_{k \sigma} \\
-\mu N_{S}\left(\frac{1}{N_{S}} \sum_{k \sigma} c_{k \sigma}^{\dagger} c_{k \sigma}-n_{c}\right)+\sum_{i} \lambda_{i} Q_{i}, \\
H_{J}(\tau)=J \sum_{i} \mathbf{S}_{f i} \cdot \mathbf{S}_{c i},
\end{gathered}
$$

with $\mathbf{S}_{c_{i}}=\Sigma_{\sigma \sigma^{\prime}} c_{i \sigma^{\dagger}}^{\dagger} \boldsymbol{\tau}_{\sigma \sigma^{\prime}} c_{i \sigma^{\prime}}$ and $\mathbf{S}_{f i}=\mathbf{S}_{i}$.

We perform a Hubbard-Stratonovich transformation on the Kondo interaction term $H_{J}(\tau)$. Since more than one field is implied in the transformation, an uncertainty is left on the way of decoupling. We propose to remove it in the following way. First, we note that $H_{J}(\tau)$ may also be written as

$$
H_{J}(\tau)=-\frac{3 J}{8} \sum_{i} n_{f c_{i}} n_{c f_{i}}+\frac{J}{2} \sum_{i} \mathbf{S}_{f c_{i}} \cdot \mathbf{S}_{c f_{i}}
$$

where $\mathbf{S}_{f c_{i}}=\Sigma_{\sigma \sigma^{\prime}} f_{i \sigma}^{\dagger} \boldsymbol{\tau}_{\sigma \sigma^{\prime}} c_{i \sigma^{\prime}}$ and $n_{f c_{i}}=\Sigma_{\sigma \sigma^{\prime}} f_{i \sigma}^{\dagger} \tau_{\sigma \sigma^{\prime}}^{0} c_{i \sigma^{\prime}}$ (respectively, $\mathbf{S}_{c f_{i}}$ and $n_{c f_{i}}$ their Hermitian conjugate).

The Kondo interaction term is then given by any linear combination of $J \sum_{i} \mathbf{S}_{f i} \cdot \mathbf{S}_{c i}$ (with a weighting factor $x$ ) and of the term appearing in the right-hand side of Eq. (4) [with a weighting factor $(1-x)]$. $x$ is chosen so as to recover the usual results obtained within the slave-boson theories. One can check that this is the case for $x=1 / 3$. The Kondo interaction term is then given by

$$
H_{J}(\tau)=J_{S} \sum_{i}\left(\mathbf{S}_{f_{i}} \cdot \mathbf{S}_{c_{i}}+\mathbf{S}_{f c_{i}} \cdot \mathbf{S}_{c f_{i}}\right)-J_{C} \sum_{i} n_{f c_{i}} n_{c f_{i}},
$$

with $J_{S}=J / 4$ and $J_{C}=J / 3$.

Performing a generalized Hubbard-Stratonovich transformation on the partition function $Z$ makes the fields $\Phi_{i}, \Phi_{i}^{*}$ (for charge) and $\boldsymbol{\xi}_{f_{i}}, \boldsymbol{\xi}_{c_{i}}$ appear (omitting the fields associated to $\mathbf{S}_{f c_{i}}, \mathbf{S}_{c f_{i}}$ ). We get

$$
Z=\int d \Phi_{i} d \Phi_{i}^{*} d \boldsymbol{\xi}_{f_{i}} d \boldsymbol{\xi}_{c_{i}} \mathcal{D} c_{i \sigma} \mathcal{D} f_{i \sigma} d \lambda_{i} \exp \left[-\int_{0}^{\beta} \mathcal{L}^{\prime}(\tau) d \tau\right]
$$




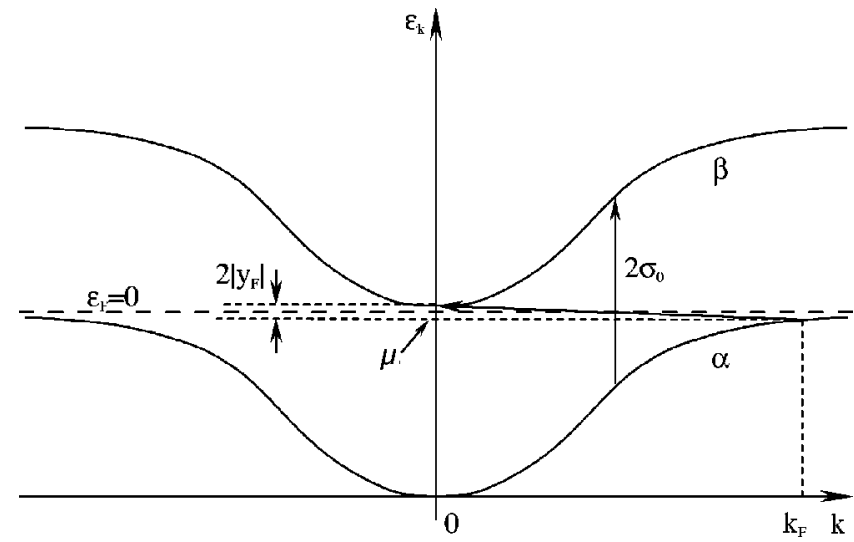

FIG. 1. Energy versus wave vector $k$ for the two bands $\alpha$ and $\beta$. Note the presence of a direct gap of value $2 \sigma_{0}$ and of an indirect gap of value $2\left|y_{F}\right|$.

with

$$
\begin{aligned}
& \mathcal{L}^{\prime}(\tau)=\mathcal{L}_{0}(\tau)+H_{0}(\tau)+H_{J}^{\prime}(\tau) \\
H_{J}^{\prime}(\tau)= & \sum_{i \sigma \sigma^{\prime}}\left(c_{i \sigma}^{\dagger} f_{i \sigma}^{\dagger}\right) \\
& \times\left(\begin{array}{cc}
-J_{S} i \boldsymbol{\xi}_{f_{i}} \cdot \boldsymbol{\tau}_{\sigma \sigma^{\prime}} & J_{C} \Phi_{i}^{*} \tau_{\sigma \sigma^{\prime}}^{0} \\
J_{C} \Phi_{i} \tau_{\sigma \sigma^{\prime}}^{0} & -J_{S} i \boldsymbol{\xi}_{c_{i}} \cdot \boldsymbol{\tau}_{\sigma \sigma^{\prime}}
\end{array}\right)\left(\begin{array}{c}
c_{i \sigma^{\prime}} \\
f_{i \sigma^{\prime}}
\end{array}\right) \\
& +J_{C} \sum_{i} \Phi_{i}^{*} \Phi_{i}+J_{S} \sum_{i} \boldsymbol{\xi}_{f_{i}} \cdot \boldsymbol{\xi}_{c_{i}} \cdot
\end{aligned}
$$

\section{A. Saddle point}

The saddle-point solution is obtained for space- and timeindependent fields $\Phi_{0}, \lambda_{0}, \xi_{f_{0}}$, and $\xi_{c_{0}}$. In the magnetically disordered regime $\left(\xi_{f_{0}}=\xi_{c_{0}}=0\right)$, it leads to renormalized bands $\alpha$ and $\beta$ as schematized in Fig. 1. Noting $\sigma_{0}^{(*)}$ $=J_{C} \Phi_{0}^{(*)}$ and $\varepsilon_{f}=\lambda_{0}, \alpha_{k \sigma}^{\dagger}|0\rangle$ and $\beta_{k \sigma}^{\dagger}|0\rangle$ are the eigenstates of

$$
\mathbf{G}_{0}^{-1 \sigma}(\mathbf{k}, \tau)=\left(\begin{array}{cc}
\partial_{\tau}+\varepsilon_{k} & \sigma_{0}^{*} \\
\sigma_{0} & \partial_{\tau}+\varepsilon_{f}
\end{array}\right),
$$

with, respectively, the eigenenergies $\left(\partial_{\tau}+E_{k}^{-}\right)$and $\left(\partial_{\tau}\right.$ $\left.+E_{k}^{+}\right)$. In the notations $x_{k}=\varepsilon_{k}-\varepsilon_{f}, y_{k}^{ \pm}=E_{k}^{ \pm}-\varepsilon_{f}$, and $\Delta_{k}$ $=\sqrt{x_{k}^{2}+4 \sigma_{0}^{2}}$, we get

$$
y_{k}^{ \pm}=\left(x_{k} \pm \Delta_{k}\right) / 2 \text {. }
$$

Let us note $U_{k \sigma}^{\dagger}$ the matrix transforming the initial basis $\left(c_{k \sigma}^{\dagger} f_{k \sigma}^{\dagger}\right)$ to the eigenbasis $\left(\alpha_{k \sigma}^{\dagger} \beta_{k \sigma}^{\dagger}\right)$. The Hamiltonian being Hermitian, the matrix $U_{k \sigma}$ is unitary: $U_{k \sigma} U_{k \sigma}^{\dagger}=U_{k \sigma}^{\dagger} U_{k \sigma}$ $=1$. In the notation

$$
U_{k \sigma}^{\dagger}=\left(\begin{array}{cc}
-v_{k} & u_{k} \\
u_{k} & v_{k}
\end{array}\right),
$$

we have

$$
\begin{aligned}
& u_{k}=\frac{-\sigma_{0} / y_{k}^{-}}{\sqrt{1+\left(\sigma_{0} / y_{k}^{-}\right)^{2}}}=\frac{1}{2}\left[1+\frac{x_{k}}{\Delta_{k}}\right], \\
& v_{k}=\frac{1}{\sqrt{1+\left(\sigma_{0} / y_{k}^{-}\right)^{2}}}=\frac{1}{2}\left[1-\frac{x_{k}}{\Delta_{k}}\right] .
\end{aligned}
$$

The saddle-point equations together with the conservation of the number of conduction electrons are written as

$$
\begin{gathered}
\sigma_{0}=\frac{1}{N_{S}} J_{C} \sum_{k \sigma} u_{k} v_{k} n_{F}\left(E_{k}^{-}\right), \\
1=\frac{1}{N_{S}} \sum_{k \sigma} u_{k}^{2} n_{F}\left(E_{k}^{-}\right), \\
n_{c}=\frac{1}{N_{S}} \sum_{k \sigma} v_{k}^{2} n_{F}\left(E_{k}^{-}\right) .
\end{gathered}
$$

Their resolution leads to

$$
\begin{gathered}
\left|y_{F}\right|=D \exp \left[-2 /\left(\rho_{0} J_{C}\right)\right], \\
2 \rho_{0} \sigma_{0}^{2} /\left|y_{F}\right|=1, \\
\mu=0
\end{gathered}
$$

where $y_{F}=\mu-\varepsilon_{F}$ and $\rho_{0}$ is the bare density of states of conduction electrons $\left(\rho_{0}=1 / 2 D\right.$ for a flat band). Noting $y$ $=E-\varepsilon_{F}$, the density of states at the energy $E$ is $\rho(E)$ $=\rho_{0}\left(1+\sigma_{0}^{2} / y^{2}\right)$. If $n_{c}<1$, the chemical potential is located just below the upper edge of the $\alpha$ band. The system is metallic. The density of states at the Fermi level is strongly enhanced towards the bare density of states of conduction electrons: $\rho\left(E_{F}\right) / \rho_{0}=\left(1+\sigma_{0}^{2} / y_{F}^{2}\right) \sim 1 /\left(2 \rho_{0}\left|y_{F}\right|\right)$. That corresponds to the flat part of the $\alpha$ band in Fig. 1. It is associated to the formation of a Kondo or Abrikosov-Suhl resonance pinned at the Fermi level resulting of the Kondo effect. The low-lying excitations are quasiparticles of large effective mass $m^{*}$ as observed in heavy-Fermion systems. Also note the presence of a hybridization gap between the $\alpha$ and the $\beta$ bands. The direct gap of value $2 \sigma_{0}$ is much larger than the indirect gap equal to $2\left|y_{F}\right|$. The saddle-point solution transposes to $N=2$ the large- $N$ results obtained within the slaveboson mean-field theories.

\section{B. Gaussian fluctuations}

We now consider the Gaussian fluctuations around the saddle-point solution. Following Read and Newns, ${ }^{17}$ we take advantage of the local U(1) gauge transformation of the Lagrangian $\mathcal{L}^{\prime}(\tau)$

$$
\begin{gathered}
\Phi_{i} \rightarrow r_{i} \exp \left(i \theta_{i}\right), \\
f_{i} \rightarrow f_{i}^{\prime} \exp \left(i \theta_{i}\right), \\
\lambda_{i} \rightarrow \lambda_{i}^{\prime}+i \partial \theta_{i} / \partial \tau .
\end{gathered}
$$

We use the radial gauge in which the modulus of both fields $\Phi_{i}$ and $\Phi_{i}^{*}$ are the radial field $r_{i}$, and their phase $\theta_{i}$ (via its time derivative) is incorporated into the Lagrange multiplier 
$\lambda_{i}$, which turns out to be a field. Use of the radial instead of the Cartesian gauge bypasses the familiar complications of infrared divergences associated with unphysical Goldstone bosons. We let the fields fluctuate away from their saddlepoint values: $r_{i}=r_{0}+\delta r_{i}, \lambda_{i}=\lambda_{0}+\delta \lambda_{i}, \boldsymbol{\xi}_{f_{i}}=\delta \boldsymbol{\xi}_{f_{i}}$, and $\boldsymbol{\xi}_{c_{i}}$ $=\delta \boldsymbol{\xi}_{c_{i}}$. After integrating out the Grassmann variables in the partition function in Eq. (6), we get

$$
Z=\int \mathcal{D} r_{i} \mathcal{D} \lambda_{i} \mathcal{D} \boldsymbol{\xi}_{f_{i}} \mathcal{D} \boldsymbol{\xi}_{c_{i}} \exp \left[-S_{e f f}\right]
$$

where the effective action is

$$
\begin{aligned}
S_{e f f}= & -\sum_{k, i \omega_{n}} \ln \operatorname{Det} \mathbf{G}^{-1}\left(\mathbf{k}, i \omega_{n}\right) \\
& +\beta\left[J_{C} \sum_{i} r_{i}^{2}+J_{S} \sum_{i} \boldsymbol{\xi}_{f_{i}} \cdot \boldsymbol{\xi}_{c_{i}}+N_{S}\left(\mu n_{c}-\lambda_{0}\right)\right],
\end{aligned}
$$

with

$$
\left[\mathbf{G}^{-1}\left(i \omega_{n}\right)\right]_{i j}^{\sigma \sigma^{\prime}}=\left(\begin{array}{cc}
{\left[\left(-i \omega_{n}-\mu\right) \delta_{i j}-t_{i j}\right] \delta_{\sigma \sigma^{\prime}}-J_{S} i \boldsymbol{\xi}_{f_{i}} \cdot \boldsymbol{\tau}_{\sigma \sigma^{\prime}} \delta_{i j}} & \left(\sigma_{0}+J_{C} \delta r_{i}\right) \delta_{\sigma \sigma^{\prime}} \delta_{i j} \\
\left(\sigma_{0}+J_{C} \delta r_{i}\right) \delta_{\sigma \sigma^{\prime}} \delta_{i j} & {\left[-i \omega_{n}+\varepsilon_{f}+\delta \lambda_{i}\right] \delta_{\sigma \sigma^{\prime}} \delta_{i j}-J_{S} i \boldsymbol{\xi}_{c_{i}} \cdot \boldsymbol{\tau}_{\sigma \sigma^{\prime}} \delta_{i j}}
\end{array}\right)
$$

Expanding up to the second order in the Bose fields, one obtains the Gaussian corrections $S_{e f f}^{(2)}$ to the saddle-point effective action

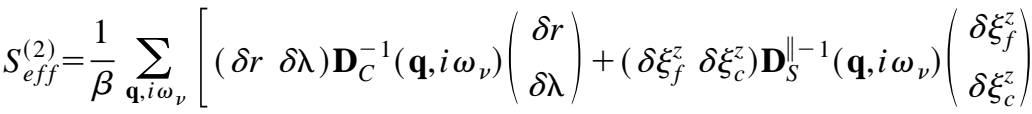

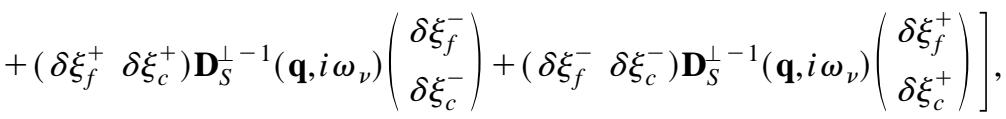

where the boson propagators split into the following charge and longitudinal spin parts

$$
\begin{gathered}
\mathbf{D}_{C}^{-1}\left(\mathbf{q}, i \omega_{\nu}\right)=\left(\begin{array}{cc}
J_{C}\left[1-J_{C}\left\{\bar{\varphi}_{2}\left(\mathbf{q}, i \omega_{\nu}\right)+\bar{\varphi}_{m}\left(\mathbf{q}, i \omega_{\nu}\right)\right\}\right] & -J_{C} \bar{\varphi}_{1}\left(\mathbf{q}, i \omega_{\nu}\right) \\
-J_{C} \bar{\varphi}_{1}\left(\mathbf{q}, i \omega_{\nu}\right) & -\bar{\varphi}_{f f}\left(\mathbf{q}, i \omega_{\nu}\right)
\end{array}\right), \\
\mathbf{D}_{S}^{\|-1}\left(\mathbf{q}, i \omega_{\nu}\right)=\left(\begin{array}{cc}
J_{S}^{2} \varphi_{f f}^{\|}\left(\mathbf{q}, i \omega_{\nu}\right) & J_{S}\left[1+J_{S} \varphi_{c f}^{\|}\left(\mathbf{q}, i \omega_{\nu}\right)\right] \\
J_{S}\left[1+J_{S} \varphi_{f c}^{\|}\left(\mathbf{q}, i \omega_{\nu}\right)\right] & J_{S}^{2} \varphi_{c c}^{\|}\left(\mathbf{q}, i \omega_{\nu}\right)
\end{array}\right)
\end{gathered}
$$

and equivalent expression for the transverse spin part $\mathbf{D}_{S}^{\perp-1}\left(\mathbf{q}, i \omega_{\nu}\right)$. The expression of the different bubbles are given in the Appendix. The charge-boson propagator $\mathbf{D}_{C}\left(\mathbf{q}, i \omega_{\nu}\right)$ associated to the Kondo effect is equivalent to that obtained in the $1 / N$-expansion theories. The originality of the approach is to simultaneously derive the spin propagator $\mathbf{D}_{S}^{\|-1}\left(\mathbf{q}_{,}, i \omega_{\nu}\right)$ and $\mathbf{D}_{S}^{\perp-1}\left(\mathbf{q}, i \omega_{\nu}\right)$ associated to the spin-fluctuation effects. Note that in the magnetically-disordered phase, the charge and spin contributions in $S_{\text {eff }}$ are totally decoupled.

\section{DYNAMICAL SPIN SUSCEPTIBILITY}

The next step is to consider the dynamical spin susceptibility. For that purpose, we study the linear response $M_{f}$ to the source term $-2 \mathbf{S}_{f} \cdot \mathbf{B}$ (we consider $\mathbf{B}$ colinear to the $\mathbf{z}$ axis). The effect on the partition function expressed in Eq. (6) is to change the Hamiltonian $H_{J}^{\prime}(\tau)$ to $H_{J}^{\prime B}(\tau)$,

$$
H_{J}^{\prime B}(\tau)=\sum_{i \sigma \sigma^{\prime}}\left(c_{i \sigma}^{\dagger} f_{i \sigma}^{\dagger}\right)\left(\begin{array}{cc}
-J_{S} i \boldsymbol{\xi}_{f_{i}} \cdot \boldsymbol{\tau}_{\sigma \sigma^{\prime}} & J_{C} \Phi_{i}^{*} \tau_{\sigma \sigma^{\prime}}^{0} \\
J_{C} \Phi_{i} \tau_{\sigma \sigma^{\prime}}^{0} & \sum_{\alpha=x, y, z}\left(-J_{S} i \xi_{c_{i}}^{\alpha}-B \delta_{\alpha z}\right) \cdot \tau_{\sigma \sigma^{\prime}}^{\alpha}
\end{array}\right)\left(\begin{array}{l}
c_{i \sigma^{\prime}} \\
f_{i \sigma^{\prime}}
\end{array}\right)+J_{C} \sum_{i} \Phi_{i}^{*} \Phi_{i}+J_{S} \sum_{i} \boldsymbol{\xi}_{f_{i}} \cdot \boldsymbol{\xi}_{c_{i}} \cdot
$$

Introducing the change of variables $\xi_{c_{i}}^{\alpha}=\xi_{c_{i}}^{\alpha}-i B / J_{S}$, we connect the $f$ magnetization and the $f f$ dynamical spin susceptibility to the Hubbard Stratonovich fields $\boldsymbol{\xi}_{f_{i}}$,

$$
\begin{gathered}
M_{f}^{z}=-\frac{1}{\beta} \frac{\partial \ln Z}{\partial B_{z}}=i\left\langle\xi_{f_{i}}^{z}\right\rangle, \\
\chi_{f f}^{\alpha \beta}=-\frac{1}{\beta} \frac{\partial^{2} \ln Z}{\partial B^{\alpha} \partial B^{\beta}}=-\left\langle\xi_{f_{i}}^{\alpha} \xi_{f_{i}}^{\beta}\right\rangle+\left\langle\xi_{f_{i}}^{\alpha}\right\rangle\left\langle\xi_{f_{i}}^{\beta}\right\rangle .
\end{gathered}
$$

Using the expression (14) for the boson propagator $\mathbf{D}_{S}^{\|-1}(\mathbf{q})$, we get for the longitudinal spin susceptibility 


$$
\chi_{f f}^{\|}\left(\mathbf{q}, i \omega_{\nu}\right)=\frac{\varphi_{f f}^{\|}\left(\mathbf{q}, i \omega_{\nu}\right)}{1-J_{S}^{2}\left[\varphi_{f f}^{\|}\left(\mathbf{q}, i \omega_{\nu}\right) \varphi_{c c}^{\|}\left(\mathbf{q}, i \omega_{\nu}\right)-\varphi_{f c}^{\| 2}\left(\mathbf{q}, i \omega_{\nu}\right)-\frac{2}{J_{S}} \varphi_{f c}^{\|}\left(\mathbf{q}, i \omega_{\nu}\right)\right]}
$$

and equivalent expression for the transverse spin susceptibility $\chi_{f f}^{\perp}\left(\mathbf{q}, i \omega_{\nu}\right)$. The diagrammatic representation of Eq. (17) is reported in Fig. 2. The different bubbles $\varphi_{f f}\left(\mathbf{q}, i \omega_{\nu}\right)$, $\varphi_{c c}\left(\mathbf{q}, i \omega_{\nu}\right)$, and $\varphi_{f c}\left(\mathbf{q}, i \omega_{\nu}\right)$ are evaluated from the expressions of the Green's functions

$$
\begin{gathered}
G_{f f}\left(\mathbf{k}, i \omega_{n}\right)=u_{k}^{2} G_{\alpha \alpha}\left(\mathbf{k}, i \omega_{n}\right)+v_{k}^{2} G_{\beta \beta}\left(\mathbf{k}, i \omega_{n}\right), \\
G_{c c}\left(\mathbf{k}, i \omega_{n}\right)=v_{k}^{2} G_{\alpha \alpha}\left(\mathbf{k}, i \omega_{n}\right)+u_{k}^{2} G_{\beta \beta}\left(\mathbf{k}, i \omega_{n}\right) \\
G_{c f}\left(\mathbf{k}, i \omega_{n}\right)=G_{f c}\left(\mathbf{k}, i \omega_{n}\right) \\
=-u_{k} v_{k}\left[G_{\alpha \alpha}\left(\mathbf{k}, i \omega_{n}\right)-G_{\beta \beta}\left(\mathbf{k}, i \omega_{n}\right)\right],
\end{gathered}
$$

where $G_{\alpha \alpha}\left(\mathbf{k}, i \omega_{n}\right)$ and $G_{\beta \beta}\left(\mathbf{k}, i \omega_{n}\right)$ are the Green's functions associated to the eigenstates $\alpha_{k \sigma}^{\dagger}|0\rangle$ and $\beta_{k \sigma}^{\dagger}|0\rangle$. In the low-frequency limit, one can easily check that the dynamical spin susceptibility may be written as

$$
\chi_{f f}\left(\mathbf{q}, i \omega_{\nu}\right)=\frac{\chi_{\alpha \alpha}\left(\mathbf{q}, i \omega_{\nu}\right)+\bar{\chi}_{\alpha \beta}\left(\mathbf{q}, i \omega_{\nu}\right)}{1-J_{S}^{2} \chi_{\alpha \alpha}\left(\mathbf{q}, i \omega_{\nu}\right) \bar{\chi}_{\alpha \beta}\left(\mathbf{q}, i \omega_{\nu}\right)}
$$

for both the longitudinal and the transverse parts.

$$
\begin{aligned}
\chi_{\alpha \alpha}\left(\mathbf{q}, i \omega_{\nu}\right)= & \frac{1}{\beta} \sum_{k} \frac{n_{F}\left(E_{k}^{-}\right)-n_{F}\left(E_{k+q}^{-}\right)}{i \omega_{\nu}-E_{k+q}^{-}+E_{k}^{-}}, \\
\bar{\chi}_{\alpha \beta}\left(\mathbf{q}, i \omega_{\nu}\right)= & \frac{1}{\beta} \sum_{k}\left(u_{k}^{2} v_{k+q}^{2}+v_{k}^{2} u_{k+q}^{2}\right) \\
& \times \frac{n_{F}\left(E_{k}^{-}\right)-n_{F}\left(E_{k+q}^{+}\right)}{i \omega_{\nu}-E_{k+q}^{+}+E_{k}^{-}} .
\end{aligned}
$$

Equation (19) constitutes the main result of the paper from which the whole physical discussion on the $\mathbf{q}$ and $\omega$ dependence of the dynamical spin susceptibility follows and comparison with experiments is made.

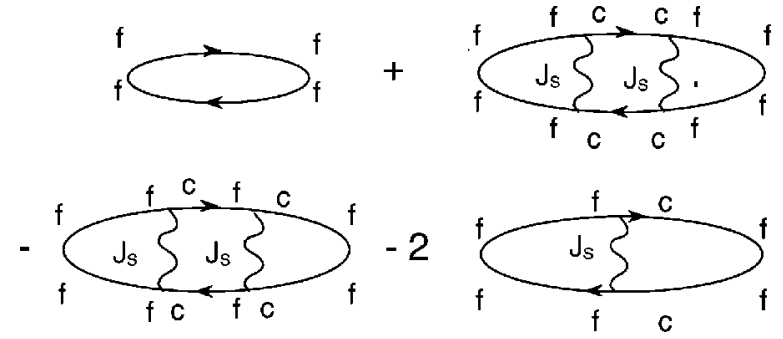

FIG. 2. Diagrammatic representation of Eq. (17) for the dynamical spin susceptibility $\chi_{f f}(\mathbf{q}, \omega)$.

\section{PHYSICAL DISCUSSION}

From Eq. (19), one can see that the dynamical spin susceptibility is made of two contributions $\chi_{\text {intra }}\left(\mathbf{q}, i \omega_{\nu}\right)$ and $\chi_{\text {inter }}\left(\mathbf{q}, i \omega_{\nu}\right)$,

$$
\chi_{f f}\left(\mathbf{q}, i \omega_{\nu}\right)=\chi_{\text {intra }}\left(\mathbf{q}, i \omega_{\nu}\right)+\chi_{\text {inter }}\left(\mathbf{q}, i \omega_{\nu}\right)
$$

with

$$
\begin{aligned}
\chi_{\text {intra }}\left(\mathbf{q}, i \omega_{\nu}\right) & =\frac{\chi_{\alpha \alpha}\left(\mathbf{q}, i \omega_{\nu}\right)}{1-J_{S}^{2} \chi_{\alpha \alpha}\left(\mathbf{q}, i \omega_{\nu}\right) \bar{\chi}_{\alpha \beta}\left(\mathbf{q}, i \omega_{\nu}\right)}, \\
\chi_{\text {inter }}\left(\mathbf{q}, i \omega_{\nu}\right) & =\frac{\bar{\chi}_{\alpha \beta}\left(\mathbf{q}, i \omega_{\nu}\right)}{1-J_{S}^{2} \chi_{\alpha \alpha}\left(\mathbf{q}, i \omega_{\nu}\right) \bar{\chi}_{\alpha \beta}\left(\mathbf{q}, i \omega_{\nu}\right)} .
\end{aligned}
$$

$\chi_{\text {intra }}\left(\mathbf{q}, i \omega_{\nu}\right)$ and $\chi_{\text {inter }}\left(\mathbf{q}, i \omega_{\nu}\right)$, respectively, represent the renormalized particle-hole pair excitations within the lower $\alpha$ band, and from the lower $\alpha$ to the upper $\beta$ band. The latter expression is reminiscent of the behavior proposed by Bernhoeft and Lonzarich ${ }^{7}$ to explain the neutron scattering observed in $\mathrm{UPt}_{3}$ with the existence of both a "slow" and a "fast" component in $\chi^{\prime \prime}(\mathbf{q}, \omega) / \omega$ due to spin-orbit coupling. Also in a phenomenological way, the same type of feature has been suggested in the duality model developed by Kuramoto and Miyake. ${ }^{27}$ To our knowledge, the proposed approach provides the first microscopic derivation from the Kondo-lattice model of such a behavior. The bare intraband susceptibility $\chi_{\alpha \alpha}(\mathbf{q}, \omega)$ is well approximated by a Lorentzian

$$
\chi_{\alpha \alpha}^{-1}(\mathbf{q}, \omega)=\rho_{\alpha \alpha}(\mathbf{q})^{-1}\left(1-i \frac{\omega}{\Gamma_{0}(\mathbf{q})}\right),
$$

where $\rho_{\alpha \alpha}=\chi_{\alpha \alpha}^{\prime}(\mathbf{q}, 0)$ and $\Gamma_{0}(\mathbf{q})$ is the relaxation rate of order $\left|y_{F}\right|=T_{K}$. This corresponds to the Lindhard continuum of the intraband particle-hole pair excitations $\chi_{\alpha \alpha}^{\prime \prime}(q, \omega) \neq 0$ as reported in Fig. 3. In the same way, we propose to schematize the low-frequency behavior $\omega \ll \omega_{0}(\mathbf{q})$ of the bare interband susceptibility by

$$
\bar{\chi}_{\alpha \beta}^{\prime,-1}(\mathbf{q}, \omega)=\rho_{\alpha \beta}(\mathbf{q})^{-1}\left[1-\frac{\omega}{\omega_{0}(\mathbf{q})}\right],
$$

where $\rho_{\alpha \beta}=\bar{\chi}_{\alpha \beta}^{\prime}(\mathbf{q}, 0)$ and $\omega_{0}(\mathbf{q})$ is a characteristic frequency scale of the interband transitions. The value of $\omega_{0}(\mathbf{q})$ is strongly structure dependent. In the simple case of a cubic band structure $\epsilon_{k}=-2 t\left(\cos k_{x}+\cos k_{y}+\cos k_{z}\right)$ (tight-binding scheme including nearest-neighbor hopping), we find a weakly wave-vector-dependent frequency around $\mathbf{q}=\mathbf{Q}$ of order of $\omega_{0}=2\left|y_{F}\right| /\left(\rho_{0} J_{C}\right)$. The latter result does not stand for more complicated band structures as obtained by de Haas-van Alphen studies ${ }^{29}$ combined with band-structure calculations in heavy fermion compounds. In the following, 


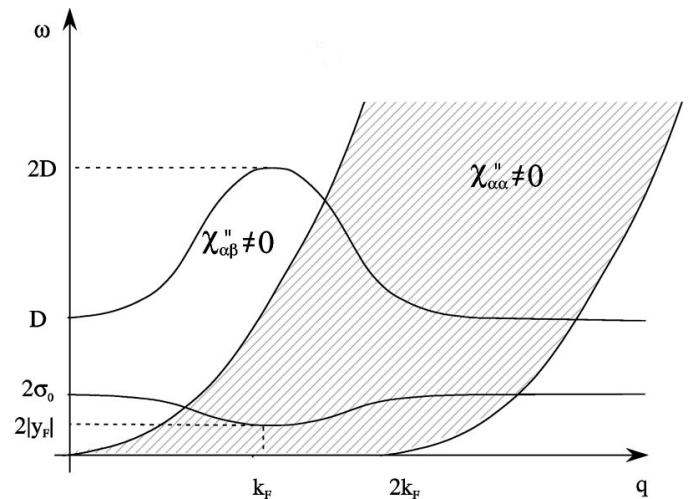

FIG. 3. Continuum of the intraband and interband electron-hole pair excitations $\chi_{\alpha \alpha}^{\prime \prime}(q, \omega) \neq 0$ and $\chi_{\alpha \beta}^{\prime \prime}(q, \omega) \neq 0$. Note the presence of a gap in the interband transitions equal to the indirect gap of value $2\left|y_{F}\right|$ at $q=k_{F}$, and to the direct gap of value $2 \sigma_{0}$ at $q=0$.

we will leave $\omega_{0}(\mathbf{q})$ as a parameter. Figure 3 reports the continuum of interband particle-hole excitations $\bar{\chi}_{\alpha \beta}^{\prime \prime} \neq 0$. Due to the presence of the hybridization gap in the density of states, the latter continuum displays a gap equal to $2 \sigma_{0}$, the value of the direct gap at $\mathbf{q}=\mathbf{0}$, and $2\left|y_{F}\right|$, the value of the indirect gap at $\mathbf{q}=\mathbf{Q}$ (close to $k_{F}$ ). More precisely, we have shown

$$
\begin{gathered}
\bar{\chi}_{\alpha \beta}^{\prime \prime}(\mathbf{0}, \omega)=4 \rho_{0} \frac{\sigma_{0}^{2}}{\omega \sqrt{\omega^{2}-4 \sigma_{0}^{2}}} \text { at } 2 \sigma_{0}<\omega<D, \\
\bar{\chi}_{\alpha \beta}^{\prime \prime}(\mathbf{Q}, \omega)=2 \rho_{0} \frac{1}{1+\omega^{2} /\left(2 \sigma_{0}\right)^{2}} \text { at } 2\left|y_{F}\right|<\omega<2 D .
\end{gathered}
$$

Far from the antiferromagnetic wave vector $\mathbf{Q}$ $=(\pi, \pi, \pi), \chi_{f f}(\mathbf{q}, \omega)$ is dominated by the intraband transitions. In the low-frequency limit, the frequency dependence of $\chi_{\text {intra }}^{\prime \prime}(\mathbf{q}, \omega)$ can be approximate to a Lorentzian

$$
\chi_{f f}^{\prime \prime}(\mathbf{q}, \omega) \approx \chi_{\text {intra }}^{\prime \prime}(\mathbf{q}, \omega)=\omega \frac{\chi_{\text {intra }}^{\prime}(\mathbf{q}) \Gamma_{\text {intra }}(\mathbf{q})}{\omega^{2}+\Gamma_{\text {intra }}(\mathbf{q})^{2}}
$$

with

$$
\begin{gathered}
\Gamma_{\text {intra }}(\mathbf{q})=\Gamma_{0}(\mathbf{q})[1-I(\mathbf{q})], \\
\chi_{\text {intra }}^{\prime}(\mathbf{q})=\frac{\rho_{\alpha \alpha}(\mathbf{q})}{(1-I(\mathbf{q}))} .
\end{gathered}
$$

$I(\mathbf{q})=J_{S}^{2} \chi_{\alpha \alpha}^{\prime}(\mathbf{q}, 0) \bar{\chi}_{\alpha \beta}^{\prime}(\mathbf{q}, 0)$. One has $\chi_{\alpha \alpha}^{\prime}(\mathbf{0}, 0)=\rho_{\alpha \alpha}(\mathbf{0})$ $=\rho\left(E_{F}\right)$ and $\chi_{\alpha \beta}^{\prime}(\mathbf{0}, 0)=\rho_{0}$. The contribution expressed in Eq. (26) is consistent with the standard Fermi-liquid theory. Note that the product $\Gamma_{\text {intra }}(\mathbf{q}) \chi_{\text {intra }}^{\prime}(\mathbf{q})=\rho_{\alpha \alpha}(\mathbf{q}) \Gamma_{0}(\mathbf{q})$ is independent of $I$.

Oppositely, at the antiferromagnetic wave vector $\mathbf{Q}$, $\chi_{f f}(\mathbf{q}, \omega)$ is driven by the interband contribution and we get

$$
\chi_{f f}^{\prime \prime}(\mathbf{Q}, \omega) \approx \chi_{\text {inter }}^{\prime \prime}(\mathbf{Q}, \omega)=\omega \frac{I \chi_{\text {inter }}^{\prime} \Gamma_{\text {inter }}}{\left(\omega-\omega_{\max }\right)^{2}+\Gamma_{\text {inter }}^{2}}
$$

with
TABLE II. Predicted values of the characteristic frequency scale $\omega_{0}$ for the interband transitions and the Stoner enhancement factor $(1-I)$ from the INS data on $\Gamma_{S S}, \Gamma_{I S}$, and $\omega_{0}$ (respectively, noted $\Gamma_{0}, \Gamma_{\text {inter }}$, and $\omega_{\max }$ in experimental papers) for the same three compounds as in Table I. Note that $\mathrm{Ce}_{1-x} \mathrm{La}_{x} \mathrm{Ru}_{2} \mathrm{Si}_{2}$ at $x=0.075$ is very close to the antiferromagnetic instability while the Stoner enhancement factor $(1-I)$ for $\mathrm{CeCu}_{6}$ is intermediate between that of the two concentrations $x=0$ and $x=0.075$ of $\mathrm{Ce}_{1-x} \mathrm{La}_{x} \mathrm{Ru}_{2} \mathrm{Si}_{2}$.

\begin{tabular}{lccccc}
\hline \hline & $\begin{array}{c}\Gamma_{0} \\
(\mathrm{meV})\end{array}$ & $\begin{array}{c}\Gamma_{\text {inter }} \\
(\mathrm{meV})\end{array}$ & $\begin{array}{c}\omega_{\max } \\
(\mathrm{meV})\end{array}$ & $\begin{array}{c}\left.\omega_{0}\right|_{\text {ded. }} \\
(\mathrm{meV})\end{array}$ & $\left.(1-I)\right|_{\text {ded. }}$ \\
\hline $\mathrm{CeCu}_{6}$ & 0.42 & 0.2 & 0.25 & 0.34 & 0.74 \\
$\mathrm{CeRu}_{2} \mathrm{Si}_{2}$ & 2.0 & 0.75 & 1.2 & 1.25 & 0.96 \\
$\mathrm{Ce}_{1-x} \mathrm{La}_{x} \mathrm{Ru}_{2} \mathrm{Si}_{2}$ & 1.4 & 0.2 & 0.2 & 1.4 & 0.14 \\
\hline \hline
\end{tabular}

$$
\begin{gathered}
\omega_{\max }=\omega_{0}(1-I), \\
\Gamma_{\text {inter }}=\omega_{0}^{2}(1-I) / \Gamma_{0}, \\
\chi_{\text {inter }}^{\prime}=\rho_{\alpha \beta} /(1-I),
\end{gathered}
$$

where $\omega_{0}, \rho_{\alpha \beta}, \Gamma_{0}$ and $I$ are the values of $\omega_{0}(\mathbf{q}), \rho_{\alpha \beta}(\mathbf{q})$, and $\Gamma_{0}(\mathbf{q})$ and $I(\mathbf{q})$ at $\mathbf{q}=\mathbf{Q}$. The role of the interband transitions have already been pointed out in previous works. ${ }^{21}$ However while the previous studies conclude to the presence of an inelastic peak at finite value of the frequency related to the hybridization gap whatever the interaction $J$ is, we emphasize that the renormalization of $\bar{\chi}_{\alpha \beta}(\mathbf{Q}, \omega)$ into $\chi_{\text {inter }}(\mathbf{Q}, \omega)$ leads to a noteworthy renormalization of the interband gap. Due to the damping introduced by intraband transitions, $\chi_{\text {inter }}^{\prime \prime}(\mathbf{Q}, \omega)$ takes a finite value at frequency much smaller than the hybridization gap. Both the relaxation rate $\Gamma_{\text {inter }}$ vanishes and the susceptibility $\chi_{\text {inter }}^{\prime}$ diverges at the antiferromagnetic transition with again the product $\Gamma_{\text {inter }} \chi_{\text {inter }}^{\prime}$ independent of $I$. Remarkably, the value $\omega_{\max }$ of the maximum of $\chi_{\text {inter }}^{\prime \prime}(\mathbf{Q}, \omega) / \omega$ is at the same time pushed to zero. Such a behavior has been effectively observed in $\mathrm{Ce}_{1-x} \mathrm{La}_{x} \mathrm{Ru}_{2} \mathrm{Si}_{2}$ (Ref. 8) with a reduction of $\Gamma_{\text {inter }}$ and $\omega_{\max }$, respectively, by a factor 4 and 6 when $x$ goes from 0 to 0.075 so when getting closer to the magnetic instability occuring at $x=0.08$. In order to make the comparison more quantitative, we propose to deduce the values of $\omega_{0}$ and (1 $-I)$ from the experimental data using the Eqs. (29): $\omega_{0}$ $=\Gamma_{0} \Gamma_{\text {inter }} / \omega_{\max }$ and $(1-I)=\omega_{\max }^{2} /\left(\Gamma_{0} \Gamma_{\text {inter }}\right)$. Table II reports the results starting from the experimental values of $\Gamma_{0}, \Gamma_{\text {inter }}, \omega_{\max }$ (respectively, noted $\Gamma_{S S}, \Gamma_{I S}, \omega_{0}$ in experimental papers) extracted from the INS results obtained in $\mathrm{CeCu}_{6}$ (Ref. 3) and $\mathrm{Ce}_{1-x} \mathrm{La}_{x} \mathrm{Ru}_{2} \mathrm{Si}_{2}$ at $x=0$ and $x=0.075$ (Ref. 8). The predictions for $\omega_{0}$ and $(1-I)$ in these compounds seem reasonable. The Stoner enhancement factor (1 $-I$ decreases in $\mathrm{Ce}_{1-x} \mathrm{La}_{x} \mathrm{Ru}_{2} \mathrm{Si}_{2}$ from $x=0$ to $x$ $=0.075$. $(1-I)$ of $\mathrm{CeCu}_{6}$ is intermediate between those two systems.

\section{CONCLUSION}

In this paper, we have set up an approach of the $S=1 / 2$ Kondo-lattice model that enlarges the standard $1 / N$ expansion theories up on the spin-fluctuation effects. The latter effects are proved to be essential for the behavior of the 
dynamical spin susceptibility near the magnetic phase transition. Our approach provides a microscopic derivation of the main features assumed in the phenomenological models of heavy Fermions as the duality model. We predict a twocomponent behavior of the dynamical spin susceptibility: a quasielastic peak typical of the Fermi-liquid excitations, and an inelastic peak at a value $\omega_{\max }$ of the frequency, which is strongly renormalized due to spin-fluctuation effects. Outstandingly well, the frequency of the inelastic peak is pushed to zero at the antiferromagnetic transition at the same time as the frequency width vanishes. The results have been compared to the inelastic neutron scattering experiment data with reasonable predictions for the Stoner enhancement factor $(1-I)$ and the characteristic frequency $\omega_{0}$ of the interband contribution to the susceptibility. Obviously, more experiments are needed for a systematic test. The issue is important since it may have implications for the quantum critical phenomena around the antiferromagnetic critical point. Work is currently in progress in that direction and will be presented in a forthcoming paper. We expect the two underlined modes to have different effects on the critical behavior with, on the one hand, the first "intraband" mode acting as a paramagnon mode as in the Hertz-Moriya-Millis theory, ${ }^{5}$ and on the other hand, additional effects brought by the second "interband" mode.

\section{ACKNOWLEDGMENTS}

We would like to thank G.G. Lonzarich, N.R. Bernhoeft, G.J. McMullan, L.P. Regnault, J. Flouquet, S. Raymond, P. Brison, and K. Miyake for very helpful discussions.

\section{APPENDIX}

The expressions of the different bubbles appearing in the expression of the boson propagators [cf. Eq. (14)] are given here (with $i=1,2, m$ or $f f$ )

$$
\begin{gathered}
\bar{\varphi}_{i}\left(\mathbf{q}, i \omega_{\nu}\right)=\varphi_{i}\left(\mathbf{q}, i \omega_{\nu}\right)+\varphi_{i}\left(-\mathbf{q},-i \omega_{\nu}\right), \\
\varphi_{1}\left(\mathbf{q}, i \omega_{\nu}\right)=-\frac{1}{\beta} \sum_{k \sigma, i \omega_{n}} G_{c f_{0}}^{\sigma}\left(\mathbf{k}+\mathbf{q}, i \omega_{n}+i \omega_{\nu}\right) G_{f f_{0}}^{\sigma}\left(\mathbf{k}, i \omega_{n}\right), \\
\varphi_{2}\left(\mathbf{q}, i \omega_{\nu}\right)=-\frac{1}{\beta} \sum_{k \sigma, i \omega_{n}} G_{c c_{0}}^{\sigma}\left(\mathbf{k}+\mathbf{q}, i \omega_{n}+i \omega_{\nu}\right) G_{f f_{0}}^{\sigma}\left(\mathbf{k}, i \omega_{n}\right), \\
\varphi_{m}\left(\mathbf{q}, i \omega_{\nu}\right)=-\frac{1}{\beta} \sum_{k \sigma, i \omega_{n}} G_{c f_{0}}^{\sigma}\left(\mathbf{k}+\mathbf{q}, i \omega_{n}+i \omega_{\nu}\right) G_{c f_{0}}^{\sigma}\left(\mathbf{k}, i \omega_{n}\right), \\
\left.\varphi_{f f}^{\|}\left(\mathbf{q}, i \omega_{\nu}\right)=-\frac{1}{\beta} \sum_{k \sigma, i \omega_{n}} G_{f f_{0}}^{\sigma}\left(\mathbf{k}+\mathbf{q}, i \omega_{n}+i \omega_{\nu}\right) G_{f f_{0}}^{\sigma}\left(\mathbf{k}, i \omega_{n}\right), i \omega_{\nu}\right)=-\frac{1}{\beta} \sum_{k \sigma, i \omega_{n}} G_{c c_{0}}^{\sigma}\left(\mathbf{k}+\mathbf{q}, i \omega_{n}+i \omega_{\nu}\right) G_{c c_{0}}^{\sigma}\left(\mathbf{k}, i \omega_{n}\right), \\
\varphi_{f c}^{\|}\left(\mathbf{q}, i \omega_{\nu}\right)=-\frac{1}{\beta} \sum_{k \sigma, i \omega_{n}} G_{f c_{0}}^{\sigma}\left(\mathbf{k}+\mathbf{q}, i \omega_{n}+i \omega_{\nu}\right) G_{f c_{0}}^{\sigma}\left(\mathbf{k}, i \omega_{n}\right), \\
\varphi_{f f}^{\perp}\left(\mathbf{q}, i \omega_{\nu}\right)=-\frac{1}{\beta} \sum_{k \sigma, i \omega_{n}} G_{f f_{0}}^{\uparrow}\left(\mathbf{k}+\mathbf{q}, i \omega_{n}+i \omega_{\nu}\right) G_{f f_{0}}^{\downarrow}\left(\mathbf{k}, i \omega_{n}\right), \\
\varphi_{c c}^{\perp}\left(\mathbf{q}, i \omega_{\nu}\right)=-\frac{1}{\beta} \sum_{k \sigma, i \omega_{n}} G_{c c_{0}}^{\uparrow}\left(\mathbf{k}+\mathbf{q}, i \omega_{n}+i \omega_{\nu}\right) G_{c c_{0}}^{\downarrow}\left(\mathbf{k}, i \omega_{n}\right), \\
\sum_{k \sigma, i \omega_{n}} G_{f c_{0}}^{\uparrow}\left(\mathbf{k}+\mathbf{q}, i \omega_{n}+i \omega_{\nu}\right) G_{f c_{0}}^{\downarrow}\left(\mathbf{k}, i \omega_{n}\right),
\end{gathered}
$$

where $G_{c c_{0}}^{\sigma}\left(\mathbf{k}, i \omega_{n}\right), G_{f f_{0}}^{\sigma}\left(\mathbf{k}, i \omega_{n}\right)$, and $G_{f c_{0}}^{\sigma}\left(\mathbf{k}, i \omega_{n}\right)$ are the Green's functions at the saddle-point level obtained by inversing the matrix $G_{0}^{\sigma}(\mathbf{k}, \tau)$ defined in Eq. (7).
*Present address: Department of Physics, MIT, Cambridge, MA 02139.

${ }^{\dagger}$ Also at the Centre National de la Recherche Scientifique (CNRS).

${ }^{1}$ H. von Löhneysen, T. Pietrus, G. Portisch, H. G. Schlager, A. Shröder, M. Sieck, and T. Trappmann, Phys. Rev. Lett. 72, 3262 (1994); H. von Löhneysen, J. Phys.: Condens. Matter 8, 9689 (1996); O. Stockert, H. v. Löhneysen, A. Schröder, M. Loewenhaupt, N. Pyka, P. L. Gammel, and U. Yaron, Physica B 230232, 247 (1997).

${ }^{2}$ S. Kambe, S. Raymond, L. P. Regnault, J. Flouquet, P. Lejay, and P. Haen, J. Phys. Soc. Jpn. 65, 3294 (1996).

${ }^{3}$ L. P. Regnault, W. A. C. Erkelens, J. Rossat-Mignod, P. Lejay, and J. Flouquet, Phys. Rev. B 38, 4481 (1988); J. RossatMignod, L. P. Regnault, J. L. Jacoud, C. Vettier, P. Lejay, J. Flouquet, E. Walker, D. Jaccard, and A. Amato, J. Magn. Magn. Mater. 76-77, 376 (1988).

${ }^{4}$ G. Aeppli, H. Yoshizawa, Y. Endoh, E. Bucher, J. Hufnagl, Y. Onuki, and T. Komatsubara, Phys. Rev. Lett. 57, 122 (1986); G. Aeppli, A. Goldman, G. Shirane, E. Bucher, and M. C. LuxSteiner, ibid. 58, 808 (1987); G. Aeppli and C. Broholm, in Handbook on the Physics and Chemistry of Rare Earths, edited by K. Gschneidner and G. H. Lander (Elsevier, New York, 1994), Vol. 19, p. 123.
${ }^{5}$ J. A. Hertz, Phys. Rev. B 14, 1165 (1976); A. J. Millis, ibid. 48, 7183 (1993); T. Moriya and T. Takimoto, J. Phys. Soc. Jpn. 64, 960 (1995).

${ }^{6}$ A. Rosch, A. Schröder, O. Stockert, and H. v. Löhneysen, Phys. Rev. Lett. 79, 159 (1997); A. Schröder, G. Aeppli, E. Bucher, R. Ramazashvili, and P. Coleman, ibid. 80, 5623 (1998).

${ }^{7}$ N. R. Bernhoeft and G. G. Lonzarich, J. Phys.: Condens. Matter 7, 7325 (1995).

${ }^{8}$ S. Raymond, L. P. Regnault, S. Kambe, J. M. Mignod, P. Lejay, and J. Flouquet, J. Low Temp. Phys. 109, 205 (1997).

${ }^{9}$ H. Tsunetsugu, M. Sigrist, and K. Ueda, Rev. Mod. Phys. 69, 809 (1997).

${ }^{10}$ S. Doniach, Physica B 91, 231 (1977).

${ }^{11}$ C. Lacroix and M. Cyrot, Phys. Rev. B 20, 1969 (1979).

${ }^{12}$ B. H. Brandow, Phys. Rev. B 33, 215 (1986).

${ }^{13}$ P. Fazekas and H. Shiba, Int. J. Mod. Phys. B 5, 289 (1991).

${ }^{14}$ T. M. Rice and K. Ueda, Phys. Rev. Lett. 55, 995 (1985).

${ }^{15}$ A. V. Goltsev, Physica B 192, 403 (1993).

${ }^{16}$ P. Coleman, Phys. Rev. B 29, 3035 (1984).

${ }^{17}$ N. Read and D. N. Newns, J. Phys. C 16, 3273 (1983).

${ }^{18}$ A. J. Millis and P. A. Lee, Phys. Rev. B 35, 3394 (1987).

${ }^{19}$ A. Auerbach and K. Levin, Phys. Rev. Lett. 57, 877 (1986). 
${ }^{20}$ M. Lavagna, A. J. Millis, and P. A. Lee, Phys. Rev. Lett. 58, 266 (1987).

${ }^{21}$ A. Auerbach, J. H. Kim, K. Levin, and M. R. Norman, Phys. Rev. Lett. 60, 623 (1988).

${ }^{22}$ A. Houghton, N. Read, and H. Won, Phys. Rev. B 37, 3782 (1988).

${ }^{23}$ S. Doniach, Phys. Rev. B 35, 1814 (1987).

${ }^{24}$ C. Lacroix, J. Magn. Magn. Mater. 100, 90 (1991).
${ }^{25}$ S. M. M. Evans, J. Phys.: Condens. Matter 3, 8441 (1991).

${ }^{26}$ S. M. M. Evans and B. Coqblin, Phys. Rev. B 43, 12790 (1991).

${ }^{27}$ Y. Kuramoto and K. Miyake, J. Phys. Soc. Jpn. 59, 2831 (1990).

${ }^{28}$ Y. Kuramoto, Solid State Commun. 63, 467 (1987).

${ }^{29}$ S. R. Julian, F. S. Tautz, G. J. McMullan, and G. G. Lonzarich, Physica B 199-200, 63 (1994); M. Takashita, H. Aoki, T. Terashima, S. Uji, K. Maezawa, R. Settai, and Y. Onuki, J. Phys. Soc. Jpn. 65, 515 (1996). 